\title{
Simulations of core convection and resulting dynamo action in rotating A-type stars
}

\author{
Matthew K. Browning ${ }^{1}$, Allan S. Brun ${ }^{2,1}$ \\ and Juri Toomre ${ }^{1}$ \\ ${ }^{1}$ JILA, University of Colorado at Boulder, 440 UCB, Boulder CO, USA 80309-0440 \\ email: matthew.browning@colorado.edu \\ ${ }^{2}$ SAp, CEA-Saclay, 91191 Gif-sur-Yvette, France
}

\begin{abstract}
We present the results of 3-D nonlinear simulations of magnetic dynamo action by core convection within A-type stars of $2 M_{\odot}$ with a range of rotation rates. We consider the inner $30 \%$ by radius of such stars, with the spherical domain thereby encompassing the convective core and a portion of the surrounding radiative envelope. The compressible NavierStokes equations, subject to the anelastic approximation, are solved to examine highly nonlinear flows that span multiple scale heights, exhibit intricate time dependence, and admit magnetic dynamo action. Small initial seed magnetic fields are found to be amplified greatly by the convective and zonal flows. The central columns of strikingly slow rotation found in some of our progenitor hydrodynamic simulations continue to be realized in some simulations to a lesser degree, with such differential rotation arising from the redistribution of angular momentum by the nonlinear convection and magnetic fields. We assess the properties of the magnetic fields thus generated, the extent of the convective penetration, the magnitude of the differential rotation, and the excitation of gravity waves within the radiative envelope.
\end{abstract}

Keywords. Convection, magnetic fields, turbulence, stars: interiors

\section{Introduction}

The observational pathologies of A-type stars have attracted scrutiny for more than a century (e.g., Maury 1897). The striking surface features seen in some of these stars are varied and extensive: abundance anomalies, strong global magnetic fields, rapid acoustic oscillations. Though many of these may be driven primarily by phenomena near the stellar surface, some could be influenced by the core convection occurring deep within the interiors of A-type stars. In particular, the long-standing puzzle regarding the origins of the surface magnetism in Ap stars raises questions about whether that core convection can sustain dynamo action, and if so, whether such interior fields could have any influences at the stellar surface or are instead buried from view.

Convection within the core clearly impacts the structure and the evolution of massive stars. Convective motions can overshoot beyond the region of superadiabatic stratification, bringing fresh fuel into the nuclear-burning core and prolonging the star's MainSequence lifetime. Observational hints at the extent of these overshooting motions have been provided through studies of best-fit isochrones to clusters (e.g., Meynet et al. 1993). Likewise some theoretical estimates have served to constrain the penetrative properties of the convection (Roxburgh 1978, 1989, 1992). Yet a detailed model of the overshooting realized from convective cores, one that gives its extent and its variation with latitude, remains lacking.

Motivated in part by these observational and theoretical challenges, we have conducted 3-D simulations of core convection deep within A-type stars. We begin by discussing 
first some properties of such nonmagnetic modelling from Browning et al. (2004), and then turn to our most recent MHD simulations. Among our aims here are to assess the penetration and overshooting achieved from convective cores, and the nature of the convective flows and the differential rotation that are established. In the calculations with magnetism, we explore whether dynamo action is realized, and if so what are the gross properties of the magnetism, its morphology, its intensity, its variations with time. Although the wealth of A-star observations lends vibrancy to our theoretical study, our work has little to say about the surface conundrums at this stage. The models here simply provide some glimpses into the complicated dynamics happening within the central regions of these stars.

\section{Approach}

Our simulations here examine the inner $30 \%$ by radius of A-type stars of $2 M_{\odot}$, rotating at the solar rate (case A) and fourfold faster (case B). The spherical domain thus encompasses the entire convective core (central $15 \%$ of star), but only a portion of the surrounding radiative envelope. We exclude the innermost $2 \%$ of the star from our computations for numerical reasons. Thus we consider turbulent convection interacting with rotation and magnetism mainly within the cores of such stars. We employ our Anelastic Spherical Harmonic (ASH) code, which solves the full 3-D anelastic MHD equations (Brun et al. 2004). The radial stratification is consistent with a 1-D stellar structure model. The MHD simulations were begun by introducing small seed dipole magnetic fields into the progenitor mature nonmagnetic simulations (Browning et al. 2004). We adopt a Prandtl number $P_{\mathrm{r}}$ of 0.25 , a magnetic Prandtl number $P_{\mathrm{m}}$ of 5 , and consider stratifications within the radiative envelope that are somewhat less stable (subadiabatic) than those of real stars. We adopt stress-free and impenetrable boundary conditions at the top and bottom of the domain, and require the magnetic field to be purely radial there. The intricate spatial variation of flows and magnetic fields in our simulations is captured by expanding both in spherical harmonics in the horizontal directions (including all degrees up to $\ell=170$ ) and in Chebyshev polynomials in the radial (employing two stacked expansion domains with $N_{\mathrm{r}}=82$ colocation points). The high-resolution modelling of the evolving turbulent convection and intricate magnetism requires the use of massively parallel supercomputers.

\section{Results}

Our simulations without magnetism (Browning et al. 2004) revealed that the convective flows within the core are vigorous and time dependent. The global connectivity allowed in the spherical domain means that the motions can couple widely separated sites, extend radially through much of the core, and span large fractions of a hemisphere. The flows penetrated into the surrounding radiative envelope, establishing a nearly-adiabatic penetrative region that was prolate in shape, having greater spatial extent near the poles than at the equator. The farther region of overshooting, where flows persisted but did not appreciably modify the prevailing stratification, had a basically spherical outer boundary. By pummelling the lower boundary of the radiative envelope, the penetrative convective plumes excited internal gravity waves within the radiative envelope.

The present MHD simulations also reveal that sustained dynamo action is realized, with the vigorous core convection serving to amplify initial seed fields by many orders of magnitude (Fig. 1). The magnetic fields that result ultimately possess energy densities comparable to that in the flows relative to the rotating frame. The magnetism is then 


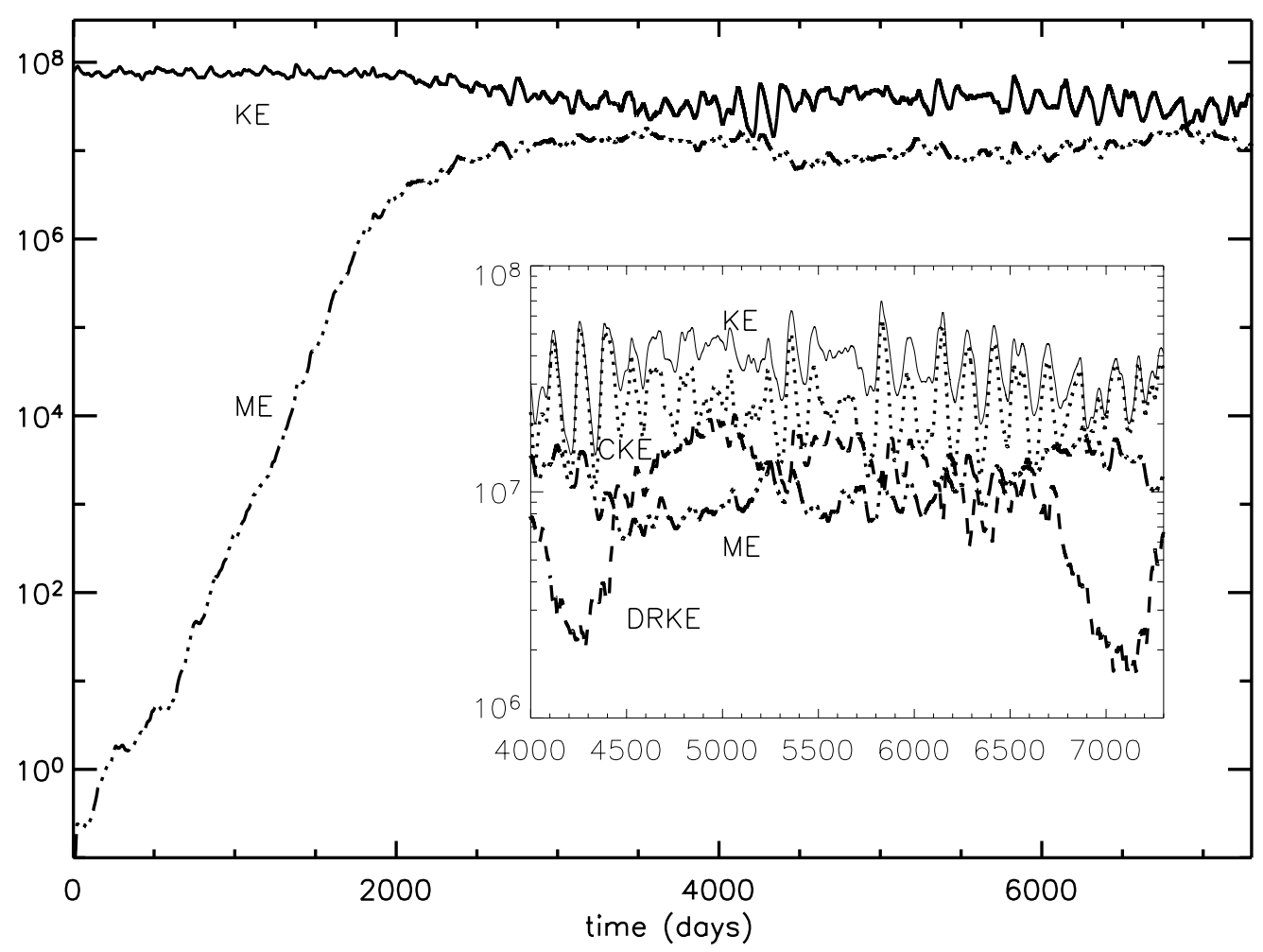

Figure 1. Temporal evolution of volume-averaged magnetic energy density (ME) from an initial seed dipole field, together with total kinetic energy density (KE). Inset: vigorous time dependence of the energy densities in the convection (CKE) and differential rotation (DRKE), with pronounced successive minima in DRKE.

sustained against decay for the multiple ohmic diffusion times that we have studied. Both simulations exhibit a rich time dependence, as magnetic fields are generated by the convective and the zonal flows and in turn feed back upon the motions, yielding the pronounced oscillatory behavior in the timetrace of energy densities (Fig. 1).

The morphologies of the flows and magnetic fields within the core are distinctive (Fig. $2)$ : the radial velocity $\left(v_{\mathrm{r}}\right)$ shows columnar alignment reminiscent of Taylor columns, whereas the magnetism is both fibril $\left(B_{\mathrm{r}}\right)$ and stretched into ribbon-like structures that extend around much of the core $\left(B_{\phi}\right)$. The differing morphologies of $v_{\mathrm{r}}$ and $B_{\mathrm{r}}$ arise partly from the smaller value of magnetic diffusivity relative to the viscous diffusivity. The organized bands of the toroidal field may be attributable to stretching by angular velocity gradients, described in mean-field dynamo theory as the $\omega$-effect, near the boundary between the core and the radiative envelope. Such stretching and amplification of the field by differential rotation also means that $B_{\phi}$ near the core boundary is only slightly diminished from its interior strength. In contrast, $v_{\mathrm{r}}$ and $B_{\mathrm{r}}$ decline rapidly in amplitude outside the convective core (Fig. $2 a, b$ ). Within the core, $B_{\mathrm{r}}$ and $B_{\phi}$ possess comparable strengths, but in the region of overshooting, where convective motions have waned, $B_{\mathrm{r}}$ has decreased by about a factor of 40 whereas $B_{\phi}$ is only roughly a factor of 3 smaller than in the core.

The magnetic fields realized within the core are predominantly fluctuating, accompanied by weak mean fields. Throughout the core, the energy associated with fluctuating 

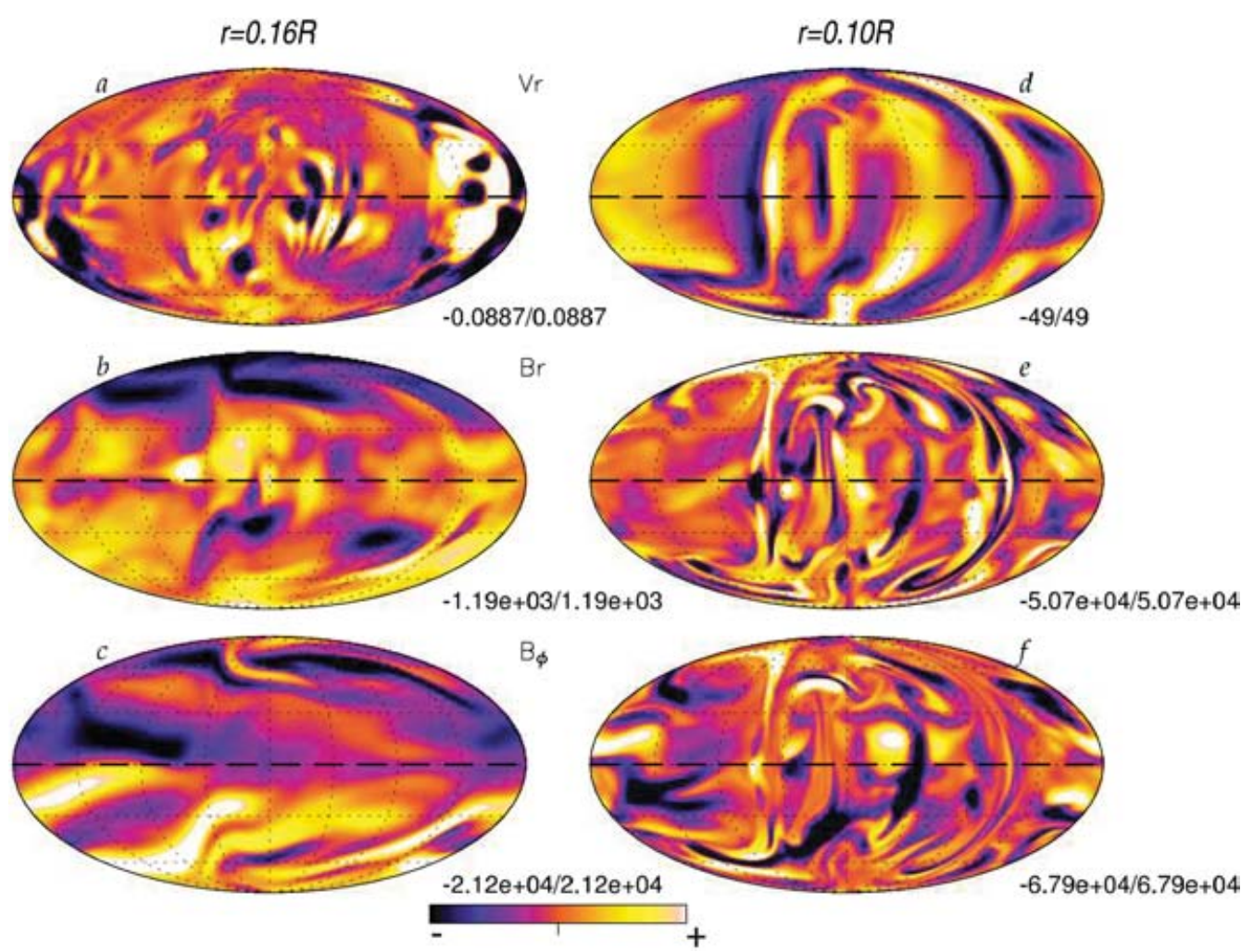

$-5.07 e+04 / 5.07 e+04$

Figure 2. Instantaneous global views for case $\mathrm{A}$ of the fields $v_{\mathrm{r}}, B_{\mathrm{r}}$, and $B_{\phi}$ on spherical surfaces (rendered as Mollweide projections) at mid-core $(r=0.10 R$, right), and in the region of overshooting $(r=0.16 R$, left). Dashed line denote the equator. Positive values are shown in bright tones, negative values in dark tones, and the ranges rendered are indicated in $\mathrm{ms}^{-1}$ and in G. Within the core, intricate magnetic fields are well-correlated with the broad convective flows, whereas outside, differential rotation stretches $B_{\phi}$ into larger-scale features.

fields accounts for more than $90 \%$ of the total magnetic energy, split in roughly equal measure between toroidal and poloidal fluctuating components. In contrast, the comparatively weak mean (axisymmetric) fields are divided unevenly between toroidal and poloidal components, with $B_{\mathrm{t}}$ stronger than $B_{\mathrm{p}}$ by about a factor of two. Outside the core, the mean toroidal field becomes the dominant component of the plummeting magnetic energy. This likely arises because the generation of fields by helical convection largely vanishes, but the stretching and amplification of toroidal fields by angular velocity contrasts persists.

The strong differential rotation established in simulations without magnetic fields by the Reynolds stresses is diminished by the presence of magnetism (Fig. 3) due to the poleward transport of angular momentum by Maxwell stresses. In case A, with ME about $40 \%$ of $\mathrm{KE}$, prominent differential rotation continues to be realized. However, in the more rapidly rotating case $\mathrm{B}$ with nearly equipartition fields, contrasts in angular velocity are greatly weakened. Case A exhibits major variations in differential rotation, with brief intervals during which the angular velocity contrasts become quite small. These grand minima in the differential rotation are visible in Figure 1 (inset) as broad dips in DKRE. The onsets of these intervals of small DRKE coincide with times when ME has grown larger than about $40 \%$ of KE, suggesting a complex interplay between strengthening magnetic fields and weakening differential rotation. 

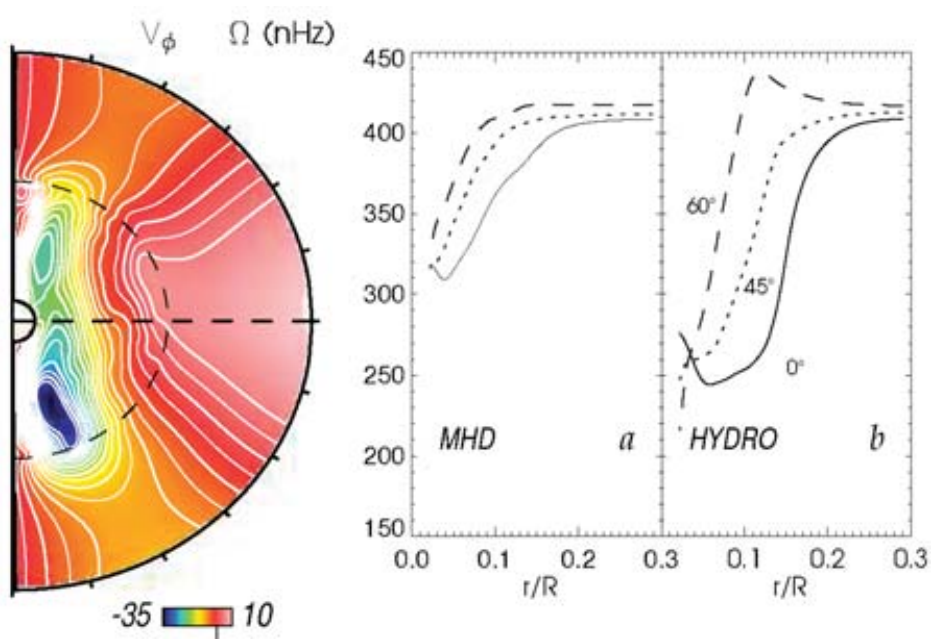

Figure 3. Differential rotation realized in case A and its hydrodynamic progenitor. (left) Contour plot in radius and latitude of longitudinal (zonal) velocity $\hat{v}_{\phi}$, with gray (color) bar and ranges (in $\mathrm{m} \mathrm{s}^{-1}$ ) indicated. (right) Angular velocity $\hat{\Omega}$ with radius for indicated latitudinal cuts in both $(a)$ case A and $(b)$ its progenitor. The magnetism from dynamo action weakens the strong angular velocity contrasts seen in the hydrodynamic simulations.

\section{Reflections}

Our simulations have revealed some striking dynamical properties of core convection deep within prototypical A-type stars. The vigorous flows penetrate into the surrounding radiative envelope, thereby exciting gravity waves, and can establish differential rotation within the core. Our MHD models further show that the flows admit magnetic dynamo action, with initial seed fields amplified by many orders of magnitude and sustained against ohmic decay. The resulting magnetic fields are mostly nonaxisymmetric and intermittent in nature, and attain strengths of up to several hundred $\mathrm{kG}$.

The generation of magnetic fields in our simulations is a complex process. Both helical convective motions and differential rotation appear to play roles in giving rise to the mean fields within the core. Thus the dynamo operating there may loosely be classified in the language of mean-field theory as being of the $\alpha^{2}-\omega$ type. The generation of the far stronger fluctuating fields is less readily parameterized in terms of such concepts, but there, too, convection and differential rotation are likely both important. Whether such differential rotation is indeed an essential element in the operation of the dynamo, and how the balance between it and the magnetism is set, remains unclear at this stage.

How the magnetic fields and flows realized in our simulations are affected by rotation, and how they are influenced by our simulation parameters, are both likely to be sensitive matters. Future work is required to assess whether the dynamos operating here are realized at all rotation rates, and if so, how the strength of the fields may scale with rotation. Also unaddressed by the present simulations is the question of whether the dynamo-generated fields can migrate to the surface where they might be observed. Magnetic buoyancy instabilities could potentially lead to such field emergence (e.g., MacGregor \& Cassinelli 2003) given strong enough magnetism, but MacDonald \& Mullan (2004) indicate difficulties with this process for realistic stratifications. The highly fibril interior fields needed for such instabilities to arise are also somewhat at odds with the large-scale magnetism observed at the surface, although the detailed morphology of fields that have risen from the core through the radiative envelope is difficult to predict. 


\section{Acknowledgements}

We thank Douglas Gough for helpful discussions, NASA for providing partial funding through SEC Theory Program grant NAG5-11879 and through the Graduate Student Researchers Program (NGT5-50416), and NSF PACI support for providing supercomputing resources at PSC, NCSA, and SDSC. Browning's travel was supported by the AAS and NSF through an International Travel Grant.

\section{References}

Browning, M.K., Brun, A.S. \& Toomre, J. 2004, ApJ, 610, 512

Brun, A.S., Miesch, M.S. \& Toomre, J. 2004, ApJ, in press

Cunha, M.S. 2002, in: C. Aerts, T.R. Bedding \& J. Christensen-Dalsgaard (eds.), Radial and Nonradial Pulsations as Probes of Stellar Physics (San Francisco: ASP), p. 272

Kochukhov, O., Bagnulo, S., Wade, G., Sangalli, L., Piskunov, N., Landstreet, J.D., Petit, P., Sigut, T.A.A. 2004, A\& A, 414, 613

MacDonald, J. \& Mullan, D.J. 2004, MNRAS, 348, 702

MacGregor, K.B. \& Cassinelli, J.P. 2003, ApJ, 586, 480

Matthews, J. 1991, PASP, 103, 5

Maury, A.C. 1897, Harvard Ann., 28, 96

Stibbs, D. 1950, MNRAS, 110, 395

Wolff, S.C. 1980, The A-Stars: Problems and Perspectives, (Washington, D.C.: NASA)

\section{Discussion}

DWORETSKY: For many years the generally accepted model for Ap star fields has been the "frozen fossil field." Does this work imply that the dynamo model for Ap stars is staging a comeback? Do I need to change my standard "Ap star" lecture slides?

Browning: No, I am not advocating a change in worldview regarding the magnetism of Ap stars. We have shown that strong fields are produced within the core, but getting those fields to the surface is another matter entirely. At this stage, we simply do not know for sure whether that is possible; modelling by MacGregor \& Cassinelli, and more recently MacDonald \& Mullan, though, suggests that it is very difficult to get the fields out.

Piskunov: Do you see a magnetic field strength maximimum at some spherical harmonics and if yes, what order of spherical harmonic is it?

BRowning: The power spectra are fairly flat until about $\ell=15$, after which they fall off rapidly. There's a modest peak at around $\ell=7$.

Moss: First, a comment to Dworetsky's question: Fossil field advocates have always recognized that the cores of A stars will host dynamos! What this work does is to provide a detailed picture which, comfortingly, agrees reasonably well with prior estimates. As the speaker said, the question is whether the field can manifest itself at the surface. So there is no need for a change in paradigm. Question: I assume that there is not a large dipole moment of the dynamo field?

Browning: The maximum amplitude of the dipole component is typically about $5 \%$ of the maximum amplitude of the total radial magnetic field. So yes, the dipole is fairly weak. 